\title{
ADAMTS mutations predict sensitivity to chemotherapy in ovarian cancer
}

Ovarian cancer is the leading cause of mortality from gynaecological cancer; however, despite aggressive treatment most patients will develop disease progression owing to chemotherapy resistance. Now, a study led by Wei Zhang suggests that the presence of mutations in the ADAMTS genes renders tumours more sensitive to platinum-based therapy.

In general, $70 \%$ of patients with ovarian cancer respond well to chemotherapy; $20 \%$ of these cases are attributable to the presence of $B R C A 1 / 2$ mutations - suggesting that other events might predict chemotherapy response, which remains highly unpredictable.

To identify these new events, Zhang and colleagues analysed the TCGA genomic and clinical data of 512 high-grade serous ovarian carcinomas. "We searched for genes that were mutated in at least two sets of chemosensitive samples, but not in any of the chemoresistant cases," explains Zhang. The investigators have reported that mutations in 8 members of the ADAMTS gene family (ADAMTS mutations) are significantly associated with improved chemotherapy sensitivity, longer overall and progression-free survival. "These findings are a useful addition to the BRCA mutation assessment for patients with ovarian cancer," says Zhang, "and we plan to characterize the molecular basis of this observation."

Of note, the identification of patients who will benefit from chemotherapy is crucial to optimize the management of ovarian cancer and in the application of a personalized medicine approach.

Alessia Errico

Original article Liu, Y. et al. Association of somatic mutations of ADAMTS genes with chemotherapy sensitivity and survival in high-grade serous ovarian carcinoma. JAMA Oncol. doi:10.1001/jamaoncol.2015.1432 